\title{
Discussion
}

\section{Transcription of live discussion: Alzheimer genes in cortical development: How do their prenatal functions relate to dementia? ${ }^{1}$}

\author{
Live Chat held 12 July 2002, 12 noon - 1 p.m. EST with Jie Shen. \\ Posted 16 September 2002. \\ Participants: Jie Shen, June Kinoshita, Kiminobu Sugaya, Richard Bowen, Keith Crutcher, Hiroko, Stavros \\ Therianos, J. Wesson Ashford
}

June Kinoshita: Jie, in your discussion text [1] you mention that you are starting to look at the results of knocking in presenilin 1 familial Alzheimer disease (PS1 FAD) mutations. Can you describe that?

Jie Shen: The experiment is still at a very early stage...

June Kinoshita: Jie, although we are all anxious to learn what effect PS1 FAD mutation knockins have, since that data are not yet ready for discussion, perhaps we can review the work on the role of PS1 on neural stem cell proliferation.

Jie Shen: PS1 is required for maintaining the proliferative state of neural progenitor cells - perhaps neural stem cells, as well.

June Kinoshita: Yes, I should have said progenitor cells.

Kiminobu Sugaya: So, without PS1, the cell differentiates? Maybe [this is] because of lack of Notch processing?

\footnotetext{
${ }^{1}$ Note: The transcript has been edited for clarity and accuracy.
}

Jie Shen: Yes, without PS1, cells differentiate through the reduction of Notch signaling.

Kiminobu Sugaya: Then PS1-knockout mice have low progenitor cells, right?

June Kinoshita: I think some previous transgenic mice with PS1 mutations were reported not to have any obvious abnormality. Were those against a background of wild-type PS1?

Jie Shen: We hope that if we can understand how PS1 regulates the cell cycle of neural progenitor cells during development, we might be able to figure out how PS1 regulates the cell cycle and fate of neural stem cells in the adult, which may be relevant to AD pathogenesis. Although PS1 is required for the regulation of the cell cycle, overexpression of PS1 may not have gain-offunction effects. Regarding neural stem cells in the adult, the problem is that there is no specific marker or promoter known to be selectively expressed in these adult neural stem cells. We are trying to get around the problem.

June Kinoshita: Are there conditional PS1-knockouts that could help answer this question? Jie, are you referring to the overexpression of mutant PS1 in transgenic mice? 
Jie Shen: Yes, most of PS1 transgenic mice are made in the wild-type PS1 background, so in these mice, there is more PS1.

Keith Crutcher: This may be completely off the wall, but I wonder if this fascinating role of PS1 in neurogenesis might suggest that mutations actually result in a hypoproliferation such that Alzheimer disease (AD) patients are actually at risk due to fewer neurons to begin with?

Jie Shen: Keith, I think it is possible. That is the reason we are looking into the knock-in mice for this.

Keith Crutcher: Has anyone actually looked at PS1 brains prior to AD (I expect there are a few) to see if this might be the case? I have wondered about whether the conclusion that AD involves significant neuronal loss might actually be due to already fewer neurons in individuals at risk ... a variant of the reserve hypothesis.

June Kinoshita: Keith, I have wondered the same thing myself. Jie, are there other approaches to gene silencing that might be applicable?

Jie Shen: June, the best approach is to have an inducible system selectively turn on and off genes in neural stem cells, but the problem is that there is no inducible system that is working as we would like, and there is no specific promoter, so we have to use other methods to get around these problems.

June Kinoshita: Jie, if the brain from a person with a PS1 FAD mutation came to autopsy, what would one look for?

Jie Shen: Keith, it is difficult to address the question in humans. By the time patients have died, fewer neurons in their brains could be due to neurodegeneration.

June Kinoshita: I think Keith is suggesting that one look at a brain of a mutation carrier who has not yet developed AD.

Keith Crutcher: Yes, June, that is what I was trying to suggest. Jie, I agree, but the evidence for neuronal degeneration is indirect, as you know. In fact, there are fewer neurons, but whether they were ever there to begin with is an open question ... I think.
Jie Shen: I do not know whether the existing technology allows accurate assessment of neuronal numbers in the brain of living patients.

Keith Crutcher: Jie, I meant postmortem analysis [of carriers who died of other causes].

June Kinoshita: There may be families with PS1 mutations who have elected to therapeutically abort a fetus ... But that is such a delicate issue.

Keith Crutcher: Jie, are pre-AD PS1 mutant carriers characterized along other neurological dimensions? I do not know this literature.

June Kinoshita: There might be someone doing volumetric measurements of such individuals, but again, I do not know how many families are being genotyped at this time.

Jie Shen: I am not aware of any literature on that. I do not think people have focused on the issue.

June Kinoshita: I wonder if Drosophila or C. elegans might be a useful model system for addressing some of these questions. What do you think, Jie?

Jie Shen: These model systems are great to address the normal function, but I am not sure whether they are good systems for FAD mutations, due to sequence divergence.

June Kinoshita: Jie, can you explain? Are there significant differences between mammalian presenilins and their homologues in fly and worm?

Jie Shen: June, yes. I do not remember the exact number, around 50 percent.

Kiminobu Sugaya: I think A $\beta$ PP production by PS mutation may also be important for stem cell differentiation or migration. We found $\mathrm{sA} \beta \mathrm{PP}$ promotes glial differentiation of stem cells. Has anybody checked on this?

Jie Shen: Kiminobu, maybe. Unfortunately, we know so little of the in vivo function of $\mathrm{A} \beta \mathrm{PP}$ and its family members in neural development. I wish someone would do what we are doing with presenilins to make double and triple cell type-specific conditional knockout to dissect out their specific function. 
June Kinoshita: For your information, Gabriel Corfas at Children's Hospital, Boston, Massachusetts, is interested in the function of $\mathrm{A} \beta \mathrm{PP}$ in neural development.

Jie Shen: Great, I should talk to him.

June Kinoshita: Regarding sequence divergence, that is intriguing in a gene that regulates progenitor cell proliferation. So, has it evolved in mammals to govern a function that is specific to mammalian brains?

Kiminobu Sugaya: Down's syndrome patient stem cells also mainly differentiated into glia rather than neurons.

Richard Bowen: I am sure many of you are aware of the work that has been done on AD and cell cycle. Mark Smith, among others, believes that neuronal death is due to aberrant entry of terminally differentiated neurons into the cell cycle [2]. Could it be that PS mutations are turning on the cell cycle in these terminally differentiated cells, resulting in dysfunction or apoptosis?

Jie Shen: PS in Drosophila may regulate cell cycle, too.

Richard Bowen: Down's syndrome patients also have much higher gonadotropin levels than does the general population. Jie, we are looking at how gonadotropins may affect the cell cycle. Since human chorionic gonadotropin hCG is extremely high during pregnancy, we are wondering if it might be affecting the presenilins.

Jie Shen: Richard, interesting.

Richard Bowen: Jie, does the PS mutation affect hormone levels in these animals?

Jie Shen: I do not know.

June Kinoshita: Jie, apropos of Richard's remark, do you have any thoughts about how presenilin might affect postmitotic neurons?

Richard Bowen: The tissue with the highest expression of PS is the testes, obviously a high rate of cell cycle there.

Kiminobu Sugaya: We have not seen any increased apoptosis in previous PS1 mutant transgenic mice.
Jie Shen: Presenilin has distinct roles in postmitotic neurons.

June Kinoshita: From what Jie writes in the discussion text, I gather that the effects of PS could be quite cell-type specific, so what happens in testes may not apply to brain, despite what we think of the gonad-brain connection!

Richard Bowen: What about cell cycle abnormalities in PS1 mutant mice?

Kiminobu Sugaya: We did not check that time.

June Kinoshita: Kiminobu, what are you referring to?

Kiminobu Sugaya: When I was with Mayo (Mayo Clinic, Jacksonville, Florida), I checked the TUNEL signal in these mice, but we did not see any increase.

J. Wesson Ashford: Another point on the testes, still wondering what the critical role of PS would be. Could there be an involvement with cell motility? That could be relevant to neurite migration.

Jie Shen: Yes, the functions of PS and Notch signaling are very context-specific. Richard, what type of experiment did Mark Smith do to show that terminally differentiated neurons can reenter the cell cycle?

Richard Bowen: He looked at the expression of cell cycle-related proteins in AD vs. control brains. Add a ref?

Jie Shen: Expression of proteins involved in the cell cycle?

Richard Bowen: Jie, yes.

Jie Shen: I am not sure about the relationship between PS and apoptosis, since we did not see a difference in TUNEL + cells between PS1-knockout and control mice. But it remains possible that at other time points, and other cell types, PS may regulate cell death.

June Kinoshita: Not to toot our own horn, but Inez Vincent put together a beautiful summary of the data on our site [3].

Jie Shen: I guess, then, the evidence is indirect, since up- or downregulation of proteins involved in cell cycle does not necessarily mean cell cycle is affected. 
Keith Crutcher: Yes; actually Mark Smith made this point [4] at the debate conference last summer, as well.

Kiminobu Sugaya: So the argument that PS1 changes a terminally differentiated neuron's cell cycle and causes neuronal cell death could be questionable.

Richard Bowen: Hiroko, Mark Smith is not the only one who has done work on this. I believe there are over 30 papers on the subject of AD and cell cycle abnormalities. I think that Karl Herrup has even shown that there is polyploidy in AD neurons, but not in control [5]. June, was there not a recent live discussion on the subject of cell cycle and AD?

June Kinoshita: Richard, yes. See the Inez Vincent reference [3].

Jie Shen: June, on the subject: Does Alzforum have the information on how to genotype patients who may have PS mutations?

June Kinoshita: Jie, patients with suspected FAD can be genotyped by Athena Diagnostics [6].

Jie Shen: Thanks, June. We have learned a lot from PS1 function and dysfunction that will help speculation on $\mathrm{AD}$ pathogenesis.

Keith Crutcher: Jie, do you have any speculation on whether normal PS1 might play a role in nonfamilial $\mathrm{AD}$, which has a much higher incidence?

June Kinoshita: For example, I am wondering if anyone has published data on PS1 gene expression or protein levels in AD brain. Someone must have.

Keith Crutcher: I do not know this literature, but I always wonder to what extent the mechanisms postulated in FAD are going to be relevant to most of AD. Jie, I think you made the argument that most PS1 mutations are likely to represent gains of function.

Richard Bowen: In the same line as Keith, does anyone know if PS1 is upregulated or downregulated in typical late-onset $\mathrm{AD}$ ?

Keith Crutcher: Jie, are you saying that the level of PS1 is reduced in sporadic AD?

Jie Shen: Keith, I am actually not sure whether FAD mutations in PS are gain-of-function. But I do think that the study of FAD mutations in PS1 will help us to understand the disease progression, in general.

Keith Crutcher: Yes, I agree. Just curious about how to relate the FAD data to sporadic AD.

Jie Shen: No, I am saying that the mechanism of neurodegeneration may be shared between PS FAD and sporadic patients.

Keith Crutcher: Yes, very possible.

Jie Shen: Actually, I think disrupted synaptic function may be the cause leading to neurodegeneration.

June Kinoshita: But what causes the disruption of synaptic function?

Keith Crutcher: Again, it would be helpful to know if PS1 mutant carriers show any differences in synaptic density.

Richard Bowen: Jie, very exciting data.

Kiminobu Sugaya: Isoe-Wada et al. [7] reported reduced PS1 expression in sporadic AD.

Jie Shen: Molecular pathways regulate synaptic function, just as PS regulates neuronal differentiation through the Notch signaling pathway.

June Kinoshita: Do you think the role of PS1 in adult brain and neurodegeneration is related to its role in progenitor cell proliferation, or some other pathway?

Jie Shen: June, maybe, it could be a contributing factor.

June Kinoshita: This suggests that there could be an impairment in the brain's repair/plasticity mechanisms.

Kiminobu Sugaya: Since we found A $\beta$ PP function in stem cell biology, I would like to speculate on PS1 effect through $\mathrm{A} \beta \mathrm{PP}$ processing, also.

Jie Shen: For example, $\mathrm{A} \beta$ disrupts synaptic function, but no one yet knows how.

June Kinoshita: What genes regulate PS1 expression? I suppose that would be one place to look in terms of understanding why PS1 expression would be reduced in sporadic AD. 
Jie Shen: Kiminobu, PS may affect downstream effects, such as synaptic function, etc., partially through their regulation of $\mathrm{A} \beta \mathrm{PP}$ processing.

Hiroko: Please, tell me, is synaptic dysfunction a key of $\mathrm{AD}, \mathrm{Jie}$ ?

Kiminobu Sugaya: In a damaged neuron, is PS1 expression increased or decreased?

Jie Shen: Hiroko, I think it may be. I meant synaptic dysfunction may be an important step leading to neurodegeneration. June, I want to address your question about brain repair, etc.

\section{June Kinoshita: Please, do.}

Jie Shen: If adult neurogenesis plays an important role in normal brain function, possibly by replenishing neuronal populations, and if FAD mutations in PS affect adult neurogenesis, then this could be a contributing mechanism for AD.

June Kinoshita: Jie, that is an interesting hypothesis, and seems as though it should be pretty testable, using some of the rodent paradigms that were used to establish the existence of adult neurogenesis.

Stavros: Concerning gene regulation, several developmental genes such as prospero in Drosophila could regulate the expression of some cell cycle-related genes. Could such regulation, using the same set of developmental "selector" genes, but in the adult, regulate cyclins as well as PS1?

Jie Shen: Possibly.

Kiminobu Sugaya: How do PS mutations affect adult neurogenesis? Decrease by increasing proliferation of the stem cells?

Jie Shen: A lot of experiments need to be done. [It could] affect proliferation or cell fate.

J. Wesson Ashford: Jie, I think that the issue in AD does not relate to neurogenesis or apoptosis, but, as asked by Hiroko, the problem is loss of synapses. The synapses are most likely lost because of disruptions of neurite function associated with neuroplasticity.

Jie Shen: Wes, I agree with you that disrupted synaptic function, loss of synapses, then loss of neurons is likely to be the main cause of $\mathrm{AD}$, but logically impaired neurogenesis could be a contributing factor, as well.

Kiminobu Sugaya: I agree. I think AD is also related to adult neurogenesis.

J. Wesson Ashford: Jie, the only problem that I can see associated with neurogenesis would be the difficulty that new neurons would have establishing new connections.

Jie Shen: I think we need to keep an open mind as to the cause of $\mathrm{AD}$, and look into all possibilities. This approach will more likely lead to the whole truth.

June Kinoshita: That is a good note on which to wrap up today. I would like to thank Jie for her participation today, and to all of the audience for attending.

\section{References}

[1] Alzheimer Research Forum, http://www.alzforum.org/res/for/ journal/shen/default.asp, 12 July 2002.

[2] A.K. Raina, X. Zhu, C.A. Rottkamp, M. Monteiro, A. Takeda and M.A. Smith, Cyclin' toward dementia: cell cycle abnormalities and abortive oncogenesis in Alzheimer disease, J Neurosci Res 61 (2000), 128-133.

[3] Alzheimer Research Forum, http://www.alzforum.org/res/for/ journal/vincent/default.asp, 7 August 2002.

[4] R. Bowser and M.A. Smith, Cell cycle proteins in Alzheimer's disease: plenty of wheels but no cycle, J Alzheimer's Disease 4 (2002), 249-254.

[5] J. Busser, D.S. Geldmacher and K. Herrup, Ectopic cell cycle proteins predict the sites of neuronal cell death in Alzheimer's disease brain, J Neurosci 18 (1998), 2801-2807.

[6] Athena Diagnostics, http://www.athenadiagnostics.com/site/ content/index.asp.

[7] K. Isoe-Wada, K. Urakami, Y. Wakutani, Y. Adachi, H. Arai, H. Sasaki and K. Nakashima, Alteration in brain presenilin1 mRNA expression in sporadic Alzheimer's disease, Eur $J$ Neurol 6 (1999), 163-167. 